\title{
Escritura colaborativa de textos en quinto grado: Razonamiento y argumentación causal sobre un fenómeno físico
}

Collaborative writing of texts in fifth grade: Causal reasoning and argumentation concerning a physical phenomenon

\section{Volumen 17, Número 1 \\ Enero-Abril}

pp. 1-25

Mario Fernando Gutiérrez R.

Revista indizada en REDALYC, $\underline{\text { SCIELO }}$

Revista distribuida en las bases de datos:

LATINDEX, DOAJ, REDIB, IRESIE, CLASE, DIALNET, SHERPA/ROMEO, QUALIS-CAPES, MIAR

Revista registrada en los directorios:

ULRICH'S, REDIE, RINACE, OEI, MAESTROTECA, PREAL, CLACSO 


\title{
Escritura colaborativa de textos en quinto grado: Razonamiento y argumentación causal sobre un fenómeno físico
}

Collaborative writing of texts in fifth grade: Causal reasoning and argumentation concerning a physical phenomenon.

\begin{abstract}
Mario Fernando Gutiérrez R. ${ }^{1}$
Resumen: Este artículo propone identificar cuáles son los cambios que se generan en los niveles de razonamiento causal y en la argumentación causal de niños de 8 a 10 años cuando escriben textos sobre el fenómeno físico de rebotar. Específicamente, se tienen como objetivos analizar la influencia de dos contextos de escritura (colaborativo e individual) sobre los niveles de razonamiento causal en la población analizada y contrastar los componentes argumentativos cuando justifican la ocurrencia de dicho fenómeno en los dos espacios. Se tuvo como hipótesis que la interacción en la situación de escritura mejoraría significativamente la calidad de los textos. Se trabajó con una muestra de cuarenta y ocho estudiantes de dos escuelas públicas de Cali (Colombia). Participaron en este estudio 19 niños y 29 niñas $(M=9.4 ; S D=0.74)$. El diseño experimental tuvo dos tipos de escritura: colaborativa e individual. En la primera condición, se conformaron triadas para la escritura de los textos; en la segunda condición, se escribieron estos de forma singular. Los sujetos experimentales fueron seleccionados y distribuidos al azar en las dos condiciones. Los resultados dan evidencia para confirmar la hipótesis del estudio. Se obtuvo un mayor número de afirmaciones causales en el contexto colaborativo y con un nivel de interacción mayor entre ellas, que las descritas en el individual. Análisis de varianza (Anova) dan cuenta de diferencias significativas en el número de variables y en el número de interacciones propuestas. En el discurso argumentativo se encontraron diferencias significativas para las afirmaciones causales, pero no para los otros componentes argumentativos. Los resultados son un indicador de que se alcanzan niveles de razonamiento más complejos escribiendo colaborativamente textos sobre fenómenos físicos que aquellos que se logran individualmente. Sin embargo, la argumentación causal no se complejiza en su estructura, más allá de aumentar el número de afirmaciones contenidas en los puntos de vista.
\end{abstract}

Palabras clave: educación primaria, escritura, razonamiento causal, argumentación causal.

\begin{abstract}
This article intends to identify which changes are generated in the levels of causal reasoning and causal argumentation in children of 8 to 10 years of age when they write texts about the physics of bounce. Specifically the study aims to analyze the influence of two contexts of writing (collaborative and individual) on levels of causal reasoning of children 8 to 10 years and contrast argumentative components when they justify the occurrence of this phenomenon in two contexts. The hypothesis was that the interaction in writing would significantly improve the quality of texts. Forty-eight students from two public schools in Cali (Colombia) participated in this study (19 boys and 29 girls) 8-10 years of age $(M=9.4 ; S D=0.74)$. The experimental design had two contexts for writing: collaborative and individual. In the first context, they formed triads for writing texts; in the second context singular texts were written. The experimental subjects were selected and were randomly distributed in the two situations. The results give evidence to confirm the hypothesis of the study. A greater number of casual statements in the collaborative context were obtained with a greater level of interaction between them, as found in under the individual context. Analysis of variance (ANOVA) account for significant differences in the number of variables and number of interactions analyzed. In the argumentative discourse significant differences for causal claims were found but not for the other argumentative components. The results are an indication that more complex levels of reasoning are reached in collaboratively writing texts about physical phenomena than those achieved individually. However, the causal argumentation are not structurally more complex, beyond increasing the number of statements.
\end{abstract}

Keywords: primary education, writing, causal reasoning, causal argumentation.

\footnotetext{
${ }_{1}$ Docente de la Pontificia Universidad Javeriana de Bogotá, Colombia.

Psicólogo, PhD. Sciences du Langage, Université Lumière (Lyon 2).
}

Dirección electrónica: mariogutierrez@javeriana.edu.co

Artículo recibido: 2 de marzo, 2016

Enviado a corrección: 26 de julio, 2016

Aprobado: 21 de noviembre, 2016 


\section{Introducción}

En este artículo se analiza la habilidad de infantes de quinto grado para escribir textos en los cuales deban argumentar causalmente sobre un fenómeno del mundo físico, bajo dos condiciones de escritura: colaborativa versus individual. En este sentido, esta propuesta se distancia de la tendencia tradicional de explorar las habilidades argumentativas de los niños y de las niñas desde la oralidad (Kuhn, 2015) y se posiciona desde el trabajo textual colaborativo y metacognitivo (Myhill y Jones, 2015). El análisis psicolingüístico de la causalidad desde una perspectiva textual evoca dos ejes conceptuales complementarios: el razonamiento causal y la argumentación causal.

El razonamiento causal es definido por Dawson y Venville (2009) como un "proceso de modelado de la situación" en el cual una persona construye un modelo mental integrador de la situación en cuestión, sus dimensiones y los factores que están involucrados. En este sentido, la causalidad forma parte de un razonamiento informal que es utilizado para resolver problemas mal estructurados, sin respuestas definitivas o correctas. Según Waismeyer, Meltzoff y Gopnik (2015), las descripciones de los eventos realizados por los adultos utilizando un lenguaje causal desempeñan un rol primordial en la comprensión de la niñez sobre la relación causa-efecto, teniendo esta comprensión una importancia primordial en las teorías sobre desarrollo cognitivo (Waismeyer, Meltzoff y Gopnik, 2015, p. 175). No obstante, como ha sido propuesto por Kim (2015, p. 132), las inferencias causales derivadas de un modelo mental no están disponibles inmediatamente de forma explícita, necesitan mediación; y es allí donde la argumentación causal tiene un rol esencial, puesto que, a través de ella, se materializa en el discurso la causalidad.

En la argumentación causal se propone una afirmación justificada en torno a la ocurrencia de un hecho o fenómeno y las razones de ello, estableciéndose así una hipótesis verificable en un sentido muy general. La estructura lingüística que pone en evidencia la relación causal es identificable intuitivamente por las personas, gracias al conocimiento que estas tienen sobre diversos esquemas argumentales específicos que son de uso cotidiano, tales como los esquemas de la causa al efecto, del efecto a la causa, de la argumentación abductiva, y de la correlación a la causa (Walton, Reed y Macagno, 2009). Para Walton (2009), los esquemas causales se estructuran alrededor de la plausibilidad, es decir, de la condición de ser aparentemente razonable, válido y creíble aquello que ha sido propuesto, y no de la probabilidad, para la cual se necesita un nivel de conocimiento más complejo y técnico. 


\section{Breve referencia teórica}

Para dar cuenta de la fundamentación teórica de esta investigación, se definirá, en primer lugar, el concepto de razonamiento causal y se enmarcará, posteriormente, dentro de la investigación sobre el aprendizaje de las ciencias y, en particular, sobre física. Se discutirá, a continuación, el nexo entre discurso argumentativo y enunciación de creencias causales, para finalizar con una revisión conceptual del núcleo del problema propuesto: la escritura colaborativa de textos argumentativos.

El razonamiento sobre la causalidad física, es decir, la apreciación de la interacción entre dos eventos en términos de las relaciones causa-efecto ha tenido un rol central en la comprensión del mundo físico (Mascalzoni, Regolin, Vallortigara y Simion, 2013). El razonamiento causal, según Atance, Metcalf, Martin-Ordas y Walker (2014), está ya presente en el razonamiento de menores de tres años sobre el mundo físico bajo el "principio de prioridad", el cual establece que las causas de las acciones o fenómenos preceden a sus consecuencias. Para estos autores hay un desfase entre la comprensión del principio de prioridad para el mundo físico y el psicológico, siendo más tardío este último.

Para Narjaikaew (2013), la investigación sobre el aprendizaje de las ciencias ha revelado que el razonamiento de los estudiantes en los cursos de esta materia se basa en ideas preexistentes sobre el mundo, y que difieren de las ideas científicas aceptadas, conociéndose como concepciones implícitas. Maskiewicz y Lineback (2013) han afirmado que la expresión "concepción implícita" ha sido ampliamente utilizada para encapsular las ideas y conceptos incorrectos que tienen los estudiantes sobre diversos fenómenos, entre ellos los físicos, y que a menudo son generalizados, estables, resistentes al cambio, y que pueden interferir con el aprendizaje. De esta forma, es válido proponerse, tal y como lo ha hecho Hardy, Kloetzer, Moelle y Sodian (2010) desde las ciencias de la educación, el saber si el estudiantado tiene la habilidad para utilizar efectivamente el discurso argumentativo para enunciar sus creencias causales sobre el mundo físico y social.

La argumentación es definida por Charaudeau (2009) como una actitud mental que consiste en describir el porqué y el cómo de los fenómenos del mundo. En esto, la argumentación se opone a los otros dos modos de organización, la descripción y la narración. Por un lado, la descripción consiste en presentar las propiedades de los seres del mundo (su naturaleza, sus características), valiéndose de diversos procedimientos de calificación. Por otro lado, la narración consiste en describir el hacer de los seres (las acciones que llevan a cabo o que sufren y los acontecimientos en los que intervienen), 
valiéndose de diversos procedimientos de puesta en escena de la narración, y permite dar cuenta del surgimiento de dichas acciones desde la intencionalidad y la temporalidad.

La naturaleza de la situación propuesta en esta investigación contempla un uso interaccional de la argumentación, pues diversas ideas se confrontan para explicar el fenómeno. Desde un punto de vista discursivo e interaccional, Plantin (2014a) define la argumentación como una actividad racional que, desarrollada a través de la lengua y dirigida a un interlocutor, intenta proveer una buena razón que hará admitir una conclusión. Para este autor, la argumentación es una actividad discursiva que contiene un razonamiento; por lo tanto, es una actividad cognitiva y epistémica vinculada con la demostración, que se desarrolla en lenguajes más formales. Dentro de las formas argumentativas ordinarias más corrientes se encuentran los argumentos de tipo causal; la relación causal en el discurso argumentativo liga una causa a sus efectos o consecuencias, tendiendo como dominio por excelencia el de las ciencias naturales (Plantin, 2014b, p. 98). Asimismo, Moeschler (2011), por su lado, afirma que el conector porque es un indicador de causalidad, pues expresa claramente la existencia de una relación entre dos fenómenos, confiriéndole un valor argumentativo a un enunciado que lo contenga.

Los estudios en argumentación oral y educación han mostrado que esta modalidad de discurso promueve y modifica de forma positiva la comprensión que se alcanza en torno a algunos objetos de conocimiento, en particular en matemática formal y en la comprensión intuitiva de fenómenos físicos. Visto que la comparación analógica facilita el cambio conceptual de un modelo mental erróneo (Gadgil, Nokes-Malach y Chi, 2012), la interacción en diálogos argumentativos produce nociones con mayor calidad y complejidad que cuando los elaboran en solitario (Kulatunga, Moog y Lewis, 2013), y mejora la comprensión conceptual (Buty y Plantin, 2008; Cavagnetto, Hand y Norton-Meier, 2009; Chin y Osborne, 2010; Erduran y Jiménez-Aleixandre, 2008; Garcia-Mila, Gilabert, Erduran y Felton, 2013; Gutiérrez y Correa, 2009; Nussbaum y Sinatra, 2003).

¿Puede identificarse esta comprensión de fenómenos físicos en la escritura colaborativa de textos argumentativos? Las tareas de escritura colaborativa pueden describirse como tareas que requieren que los estudiantes trabajen en parejas o grupos pequeños para producir conjuntamente un texto (Swain, 2001). Los estudiantes trabajan juntos durante el proceso entero de la escritura, compartiendo la autoría y la responsabilidad del producto final. La copropiedad del texto final, según Storch (2011), es el rasgo definitorio de la escritura colaborativa. 
Los estudios realizados sobre los efectos de la escritura colaborativa se han enfocado esencialmente en el aprendizaje de una lengua extranjera (English for Academic Purposes o EAP, por sus siglas en inglés), mostrando en general que el rendimiento del estudiantado mejora considerablemente, al igual que sus productos. Los textos están mejor estructurados gramaticalmente (Wigglesworth y Storch, 2009), tienen mejor contenido, una mayor organización y un vocabulario más complejo (Shehadeh, 2012), además poseen una planeación más elaborada que los textos individuales (Elola y Oskoz, 2010; Neumann y McDonough, 2015), y se convierte en una práctica más valorada que la escritura individual (Dobao y Blum, 2013).

Según Dobao (2012), la escritura colaborativa, ya sea en parejas o en pequeños grupos, permite una mayor precisión lexical y gramatical en textos escritos por estudiantes cuando aprenden en lengua extranjera (inglés); aunque la escritura en grupo ofrece menos oportunidades de participación individual, esta tiene un impacto positivo en el diálogo colaborativo. El autor afirma que los discentes trabajando en grupos pequeños prestan más atención al lenguaje y son más exitosos en la solución de problemas relacionados con este que quienes trabajan en pares. De tal forma, los estudiantes en grados básicos pueden desarrollar destrezas en escritura justamente a través de procesos de escritura colaborativa, pues estos demandan una responsabilidad compartida, promoviendo la toma de decisiones en torno al contenido, estructura y recursos lingüísticos utilizados (Storch, 2005, p. 153).

Según Neumann y McDonough (2015), la inclusión de las actividades colaborativas en las aula se ha justificado a través de la teoría sociocultural, en particular con las propuestas de Vygotsky (1979), quien afirmó que el discurso es una parte esencial del desarrollo cognitivo humano, y que, a través de este, se desarrollan diversas destrezas mediante la interacción con las demás personas y con el mundo físico. La interacción juega un papel esencial en la construcción de conocimiento mediante la creación de oportunidades para el estudiantado de obtener ayuda de expertos o, simplemente, articular pasos en el proceso de resolución de problemas a través del habla interna o externa. Según estos autores, su investigación en escritura colaborativa ha mostrado que las situaciones en las cuales los estudiantes discuten sobre el texto, antes de escribirlo, mejora el lenguaje, el contenido y la organización de estos, mejorando, así, ostensiblemente la calidad del escrito. Esto los lleva a preguntarse entonces, por qué este tipo de actividades no son utilizadas comúnmente en el aula de clase o en otros dominios de conocimiento diferentes al del aprendizaje de una segunda lengua. 
La efectividad del aprendizaje en grupos pequeños ha sido objeto de amplios estudios en la educación postsecundaria (Berland y Lee, 2012; Gosser, Kampmeier y Varma-Nelson, 2010, Kulatunga, Moog y Lewis, 2013), pero siempre dentro del discurso oral y ninguno desde la escritura de textos científicos. Se ha demostrado que la interacción mejora el rendimiento de los estudiantes cuando se aprende una segunda lengua, pero, ¿ocurre esto también en la escritura de textos sobre ciencia? En la presente investigación se abordó la capacidad de niños y niñas de quinto grado para escribir colaborativamente textos relacionados con la comprensión intuitiva del fenómeno del rebote, producido por objetos al caer. Se tuvo como objetivos identificar los niveles del razonamiento causal sobre el fenómeno físico analizado en textos escritos bajo dos condiciones experimentales (escritura colaborativa e individual), y determinar los componentes argumentativos que configuraban los enunciados en dichos textos. Esta escogencia de la escritura colaborativa tiene como fin utilizar el diálogo y la interacción entre pares para mejorar la escritura de los textos.

Entre los modos de organización textual se ha escogido el argumentativo, pues la influencia del trabajo colaborativo sobre los textos no debería limitarse a los aspectos lexicales que han sido abordados en los estudios sobre aprendizaje de una lengua extranjera. Desde la psicolingüística y según Mercier y Sperber (2011), la argumentación es un proceso discursivo que se caracteriza por manipular explícitamente representaciones en la memoria de trabajo, con el fin de generar nuevas inferencias. Por lo tanto, es la hipotética reflexión inferencial que promueve el trabajo colaborativo, y que está plasmada en los textos, el objeto de análisis de este artículo.

\section{Marco metodológico}

\subsection{Diseño experimental}

El diseño experimental incluyó trasformaciones sobre el contexto de escritura. Inicialmente, se estableció un muestreo no probabilístico, denominado por Coolican (2005) como muestreo por conveniencia, debido a que la muestra se limitó a las instituciones participantes en la presente investigación. Siguiendo las directrices de Coolican, el diseño propuso la disminución de variables alternas al experimento estableciendo primeramente una selección al azar de los posibles sujetos participantes (48 estudiantes) entre el total de estudiantes de ambas escuelas (68 estudiantes) que cumplían los criterios de participación (edad, grado y consentimiento informado). La muestra seleccionada fue asignada al azar en las dos condiciones experimentales (grupo experimental y grupo control). El grupo 1 que 
operacionaliza la variable estudiada (escritura colaborativa) y el grupo 2 que ejerce como grupo control pues no experimentó cambios en su forma habitual de trabajo (escritura individual), generando así una línea base de comparación (Coolican, 2005, p. 40). Todos los participantes discutieron de manera previa las características de un texto argumentativo con su docente, en un curso habitual de su pensum académico, específicamente el de español. En la primera condición, 12 grupos de tres estudiantes escribieron conjuntamente un texto argumentativo. En la segunda condición, 12 estudiantes lo escribieron individualmente.

\subsection{Población y muestra}

Cuarenta y ocho estudiantes participaron en este estudio (19 niños y 29 niñas), de 8 a 10 años de edad $(M=9.4 ; S D=0.74)$. Fueron seleccionados al azar en dos escuelas públicas urbanas de Cali (Colombia), las cuales están ubicadas en zonas de estrato socioeconómico medio, según la clasificación local hecha por una empresa pública del Estado colombiano. Todos tenían el español como lengua materna y ninguno era bilingüe. En el momento de las entrevistas cursaban el grado quinto de educación básica primaria. El número de participantes en el grupo de escritura colaborativa fue de 36 , y de 12 en el grupo de escritura individual. Siendo consistente esta investigación con las directrices del código de deontología y bioética del ejercicio de la psicología en Colombia (Colegio Colombiano de Psicólogos, COLPSIC, 2009), el cual establece los principios que deben guiar el trabajo con humanos, todos los sujetos participaron después de haberse obtenido el consentimiento informado de sus respectivos padres de familia. Este proyecto fue revisado éticamente y aprobado por profesionales del Grupo de Investigación ICAR (UMR 5191 - CNRS) de la Université Lumière (Lyon 2).

\subsection{Procedimiento}

La situación de escritura se desarrolló en una sola sesión. En la condición colaborativa se pidió a los sujetos que escribieran un texto en el cual argumentaran las razones de la ocurrencia del fenómeno del rebote de los cuerpos después de experimentar una caída libre. Estos fueron autónomos en las decisiones que han de tomarse en la escritura de cualquier texto, ellos mismos decidieron qué escribir y cómo hacerlo. Ninguna guía explícita, esquema o esbozo fue dado para la ejecución de la tarea propuesta ni se impuso un límite de tiempo. 
Se trabajó con un grupo a la vez. Los participantes no tuvieron un límite de tiempo. Se evitó la interacción entre los participantes y el entrevistador, limitándose este a responder sus preguntas en torno a los objetivos y productos esperados de la situación.

El trabajo con el grupo control fue similar. Cada estudiante fue entrevistado individualmente por el investigador en un espacio privado ajeno al salón de clase. Se utilizó la misma consigna utilizada con el grupo experimental, pero corregida en singular.

\subsection{Categorías de análisis}

Tal y como ha sido propuesto por Pereira (2011), los métodos mixtos suponen una serie de fases que se complementan (secuencial o simultáneamente) buscando obtener un conocimiento más amplio y profundo acerca del objeto de estudio para comprenderlo integralmente. Esta investigación contempló el uso de un método mixto con un estatus no dominante y de orden secuencial (Pereira, 2011); en este sentido, un método cualitativo se utilizó en una fase inicial de la investigación y uno cuantitativo en una fase posterior. Con respecto al razonamiento causal, se construyó inicialmente una escala cualitativa de siete niveles (ver Cuadro 1) a partir de un análisis inductivo realizado sobre el corpus recopilado en esta investigación. Posteriormente, se identificaron y cuantificaron las afirmaciones causales en cada texto, las variables físicas propuestas y su posible interacción.

\section{Cuadro 1}

Niveles de razonamiento en afirmaciones causales

\begin{tabular}{|c|c|}
\hline $\begin{array}{l}\text { Nivel de } \\
\text { razonamiento } \\
\text { causal }\end{array}$ & Características \\
\hline Nivel 1 & $\begin{array}{l}\text { Contiene algunas características físicas de objetos que no tienen rasgos en } \\
\text { común, sin comparación entre estos y sin factores comunes que los agrupen. }\end{array}$ \\
\hline Nivel 2 & $\begin{array}{l}\text { Contiene algunas características físicas de objetos que tienen rasgos en } \\
\text { común, comparándolos entre ellos y limitándose a los factores que los } \\
\text { agrupan. }\end{array}$ \\
\hline Nivel 3 & $\begin{array}{l}\text { Contiene las consecuencias de la colisión de un objeto, incluyendo } \\
\text { declaraciones sobre los efectos producidos cuando los objetos entran en } \\
\text { colisión contra una superficie cualquiera. }\end{array}$ \\
\hline Nivel 4 & $\begin{array}{l}\text { Contiene situaciones hipotéticas o frases condicionales que explican la } \\
\text { aparición del fenómeno propuesto. }\end{array}$ \\
\hline Nivel 5 & Contiene una única variable como origen del fenómeno propuesto. \\
\hline Nivel 6 & $\begin{array}{l}\text { Contiene causas diversas, pero no simultáneas; estas incluyen declaraciones } \\
\text { que reflejan diferentes situaciones, que son el origen del fenómeno } \\
\text { propuesto, sin especificar que ellas trabajan interactivamente. }\end{array}$ \\
\hline Nivel 7 & $\begin{array}{l}\text { Contiene causas diversas y simultáneas que son el origen del evento y } \\
\text { proponen que dichas causas trabajan de manera interactiva. }\end{array}$ \\
\hline
\end{tabular}

Fuente: Elaboración propia. 
Con respecto a la argumentación causal, se realizó un análisis de enunciados en el texto a través de un sistema de categorización para componentes argumentativos (Gutiérrez y Correa, 2009; Leitão, 2007; Sadler, 1993), este indaga la presencia de una introducción, en la cual se involucra una de un tópico de discusión; de puntos de vista, donde se incluyen las afirmaciones causales que explican el fenómeno analizado; de justificaciones, que apoyan las afirmaciones; de contra-argumentos, que pueden contener una refutación o diversas refutaciones; y de conclusiones.

\section{Resultados}

Se encontró, en los textos, causas al fenómeno discutido que estaban en relación con los conocimientos socialmente aceptados en física. El $75 \%$ de los textos en el contexto grupal y el $91.67 \%$ en el contexto individual contuvieron solo argumentos coherentes con el dominio de conocimientos de la física clásica demandado en la consigna; la diferencia la generó la introducción de un tópico contextual por parte de los grupos en la versión colaborativa, el cual ambientaba con una historia la ocurrencia de la caída de los cuerpos. Se obtuvo un total de 99 afirmaciones causales en 24 textos analizados; 76 afirmaciones causales en el contexto grupal (76.76 \%) y 33 en el contexto individual (33.33 \%). La media es de 6.33 de afirmaciones causales por texto dentro del contexto grupal y de 2.75 en el contexto individual.

Los promedios por niveles del razonamiento causal son presentados a través del Gráfico 1. 


\section{Gráfico 1}

Promedio de los niveles de razonamiento causal por contexto de escritura

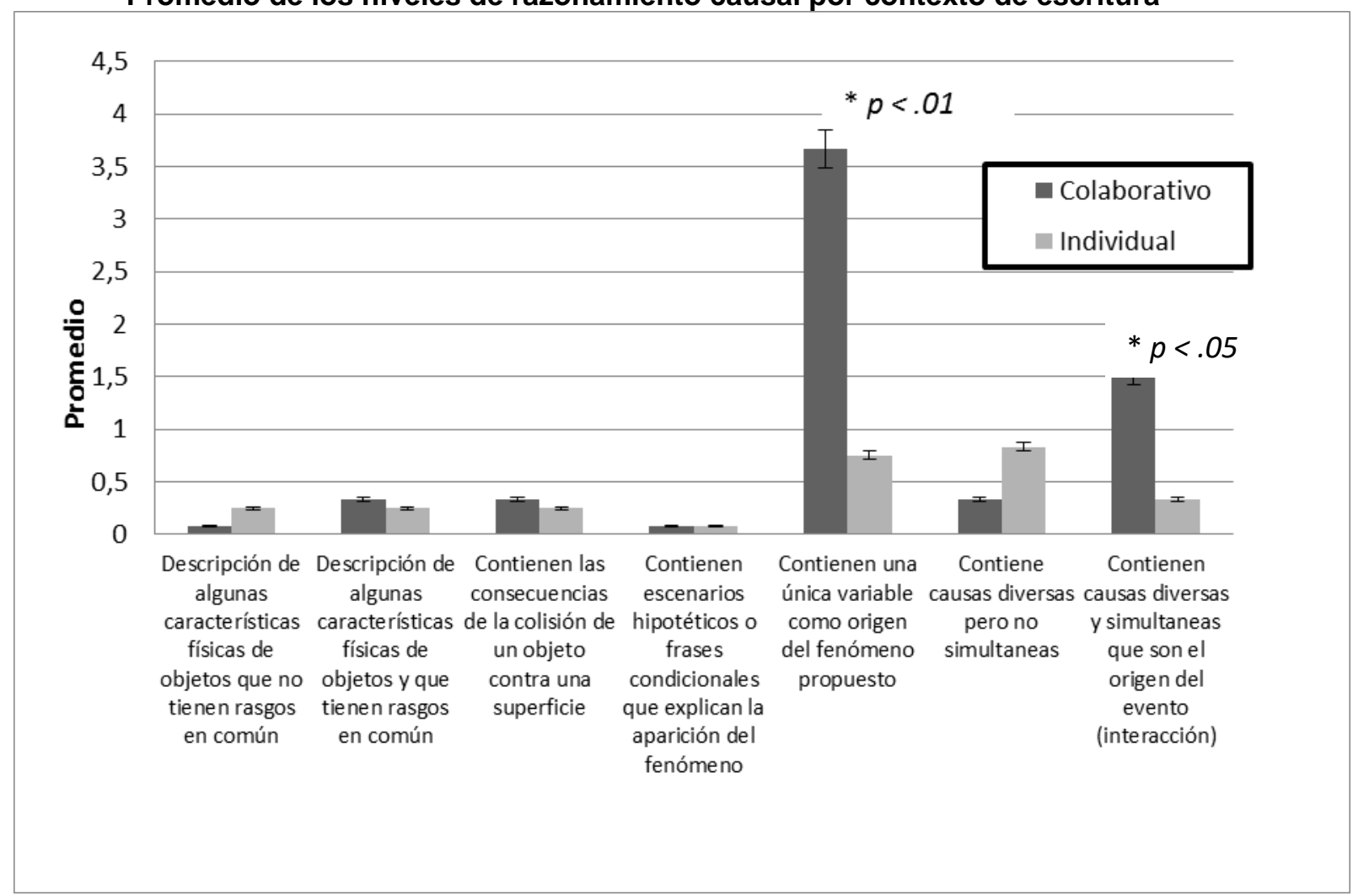

Fuente: Elaboración propia.

Se realizó un Anova encontrando que el contexto de escritura afecta la producción escrita del grupo de estudiantes de quinto grado, siendo significativamente mayor en la condición grupal $F(4,30)=25,33, p<.01$. La relación entre escritura y producción es significativa en dos variables: los estudiantes aumentan el número de causas y los hechos que les permiten explicar e interpretar el fenómeno físico propuesto, y contemplan una mayor interacción entre las variables. La interacción entre contexto y desempeño escrito se revela significativa en las afirmaciones causales de nivel cinco $F(5.41)=19.73, p<.01$. y nivel 7 , $F(4.38)=4.86, \mathrm{p}<.05$. El trabajo colaborativo permite a los estudiantes ampliar el número de variables presentes en sus textos y proponer una interacción entre las variables.

En contravía de lo hallado para las afirmaciones causales de nivel 5 y 7, no se encontraron diferencias significativas en los demás niveles: nivel $1, F(2,15)=0.51, p>.05$; nivel 2, $F(1.0)=0.37, \mathrm{p}>.05$; nivel $3, F(1.0)=0.37, \mathrm{p}>.05$; nivel $4, F(4.30)=0.07, \mathrm{p}>.05$; $\mathrm{y}$ nivel $6, F(2.51)=1.87, \mathrm{p}>.05$. El $72.5 \%$ de las afirmaciones causales del contexto grupal y el $84.6 \%$ de estas en el contexto individual contienen una sola variable explicativa.

Las variables causales son detalladas en la Tabla 1. 
Tabla 1

Porcentaje de las afirmaciones causales en función del contexto de escritura

\begin{tabular}{|c|c|c|c|}
\hline $\begin{array}{c}\text { Número } \\
\text { de } \\
\text { variables }\end{array}$ & Variable & $\begin{array}{c}\text { Contexto } \\
\text { Grupal (\%) } \\
N=76\end{array}$ & $\begin{array}{l}\text { Contexto } \\
\text { Individual } \\
\text { (\%) } \mathrm{N}=33\end{array}$ \\
\hline \multirow[t]{9}{*}{ Una } & Material de construcción & $30.4 \%$ & $18.2 \%$ \\
\hline & Peso & $28.4 \%$ & $9 \%$ \\
\hline & Presencia de un gas en su interior & $8.5 \%$ & $21-2 \%$ \\
\hline & Forma & $2.6 \%$ & $15.2 \%$ \\
\hline & Gravedad & & $3.0 \%$ \\
\hline & Tenencia de resortes & $2.6 \%$ & $3.0 \%$ \\
\hline & Tamaño & & $6.0 \%$ \\
\hline & Impulso por una causa externa & & $3.0 \%$ \\
\hline & Impacto contra otro objeto & & $6.0 \%$ \\
\hline \multirow[t]{9}{*}{ Dos } & $\begin{array}{l}\text { Material de construcción y tenencia de un gas en el } \\
\text { interior }\end{array}$ & $4.6 \%$ & $3.0 \%$ \\
\hline & Material de construcción y forma & $5.4 \%$ & \\
\hline & Tenencia de un gas en el interior y peso & & $3.0 \%$ \\
\hline & Tenencia de un gas en el interior y gravedad & $3.4 \%$ & \\
\hline & Forma del objeto y peso & $8.9 \%$ & $3.0 \%$ \\
\hline & Material de construcción y peso & $1.3 \%$ & \\
\hline & Tamaño y peso & $2.6 \%$ & $3.0 \%$ \\
\hline & Resistencia a la presión y peso & $1.3 \%$ & $3.0 \%$ \\
\hline & & $100 \%$ & $100 \%$ \\
\hline
\end{tabular}

Fuente: Elaboración propia.

Se hallaron dos tipos de respuestas en los textos, un primer tipo que contenía causas que eran susceptibles de ser percibidas en su medioambiente natural, particularmente referidas a características morfológicas prototípicas de las esferas, ejemplo: rebotan porque son redondas. Un segundo tipo conllevó una combinación arbitraria entre hechos observables, conocimientos propios de la escuela, y conceptos en proceso de aprendizaje, ejemplo rebotan porque son redondas y tienen gravedad.

Los resultados con respecto a los componentes argumentativos por contexto son presentados en la Gráfica 2. 


\section{Gráfica 2}

Promedio de componentes argumentativos por contexto de escritura

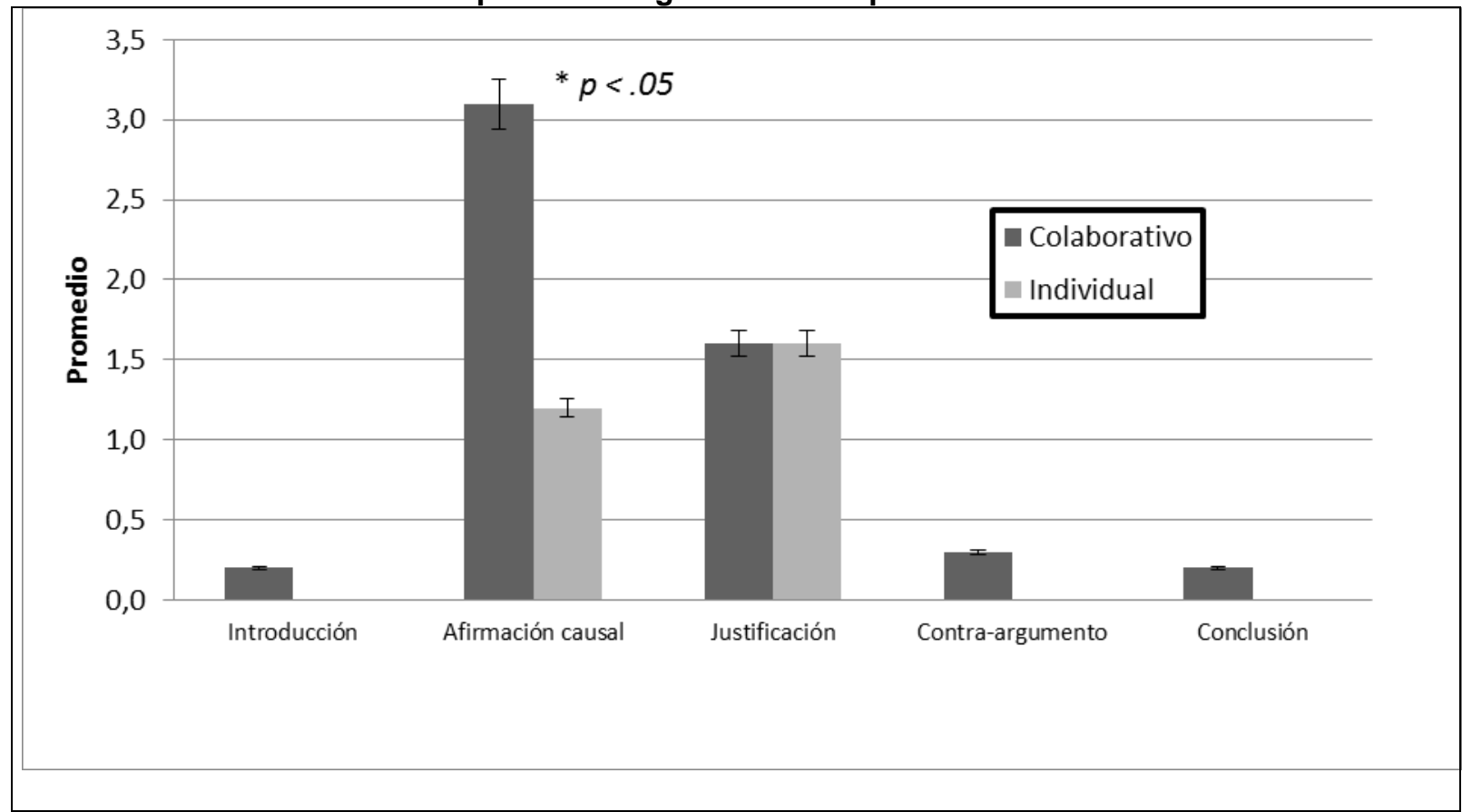

Fuente: Elaboración propia.

Se realizó un Anova encontrando que el contexto de escritura afecta un componente de la configuración argumentativa de los textos. La relación entre contexto de escritura y producción textual es significativa en las afirmaciones causales $F(7.35)=15.0 p<.05$. El trabajo colaborativo permite a los estudiantes ampliar el número de afirmaciones causales sobre las variables que afectan el fenómeno físico discutido y sus interacciones. No se encontraron diferencias significativas en los demás componentes argumentativos: introducción $F(2.20)=0.7, p>.05$; justificación $F(3.66)=0.37, p>.05$; contra-argumentos $F(3.66)=0.37, p>.05 ; y$ conclusiones $F(2.20)=0.16, p>.05$.

Una muestra de este tipo de textos se presenta en el Cuadro 2. 


\section{Cuadro 2}

Texto producido en situación colaborativa vs. individual Contexto colaborativo Contexto Individual

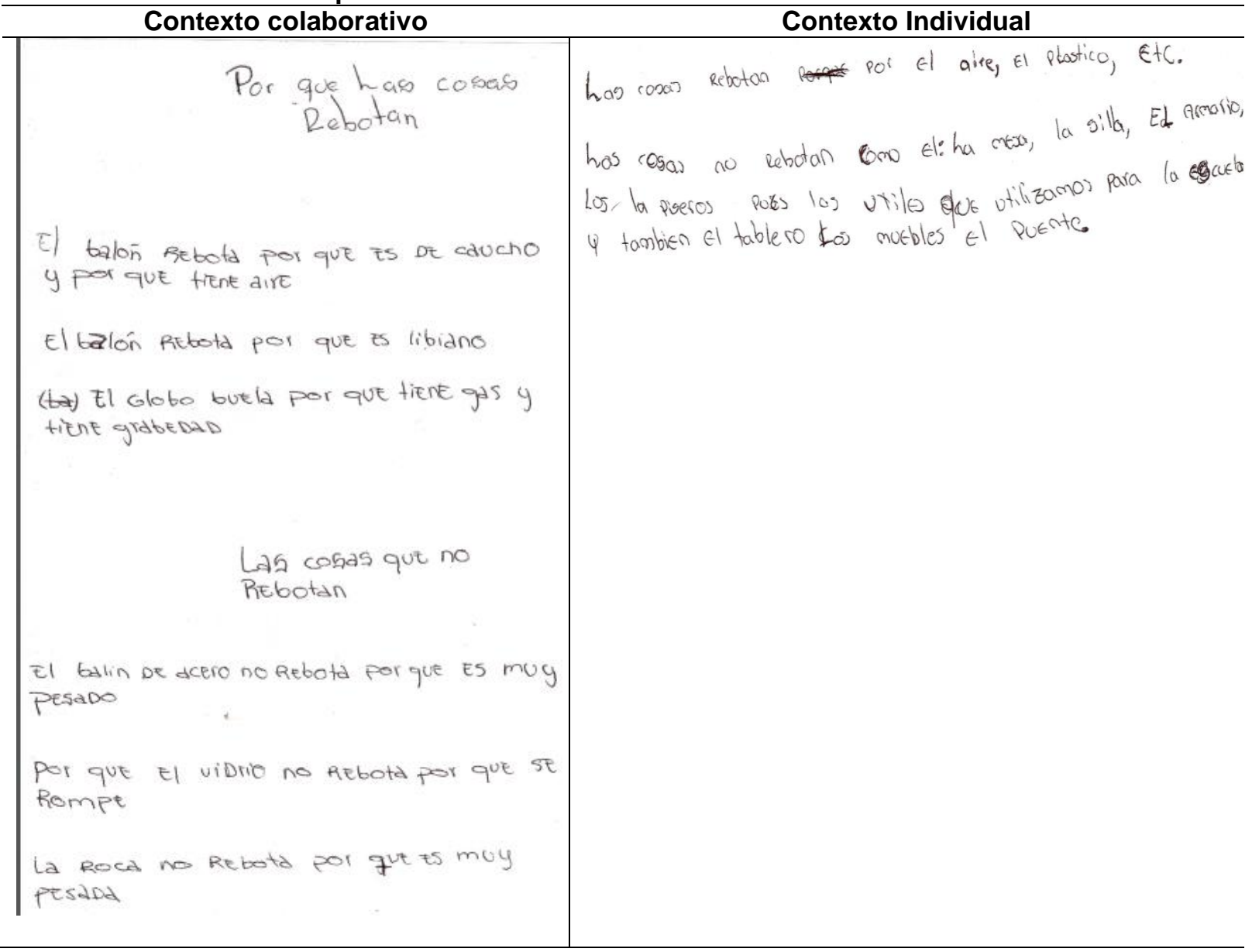

Fuente: Elaboración propia.

Esta disposición de los componentes estructurales de un argumento da cuenta generalmente de una estructura argumentativa en la cual los textos presentan las afirmaciones directamente, sin introducir al lector en la problemática planteada. Se comienza con una afirmación seguida de una justificación o de una secuencia de justificaciones que denota lo que Weisser (2004) ha llamado "serie de enunciados empilados", donde los agentes de las acciones no son identificados con precisión. Para este autor, este tipo de textos se originan en la falta de incorporación de marcadores polifónicos en los textos (Weisser, 2004, p. 3); tales como una utilización de pronombres o un tipo de elemento designativo que especifique un referente en el texto. 


\section{Discusión}

El objetivo principal de esta experiencia fue analizar la capacidad de niños de quinto grado para escribir textos relacionados con la comprensión intuitiva del fenómeno del rebote de los cuerpos al caer, cuando los escriben bajo dos condiciones: colaborativa e individual. En la escritura colaborativa se produjeron textos con un mayor número de atribuciones causales, y con mayor interacción entre ellas, que en la condición individual. La interacción discursiva entre los sujetos cuando resuelven la tarea puede ser el factor primordial para generar un mayor acopio de razones explicativas al fenómeno propuesto. El hecho de que el estudiantado en la situación propuesta por esta investigación hayan podido describir en sus textos, con un cierto nivel de detalle, las posibles causas del fenómeno propuesto, sin acompañarlos de información irrelevante, muestra como ha sido capaz de modular su estilo de escritura y darle las características de pertinencia y objetividad que demanda un texto científico.

La escritura de textos permite además identificar por parte del docente las diversas creencias que desarrolla el cuerpo estudiantil sobre los fenómenos, explicitando así las relaciones causales que este alcanza a identificar en la ocurrencia del fenómeno. Esto es consistente con la propuesta de McCormack, Bramley, Frosch, Patrick y Lagnado (2016), quienes afirman que, en el aprendizaje sobre el mundo físico, lo que está en cuestión es, a menudo, no solo si una variable específica tiene un particular poder causal, sino también la estructura de las relaciones causales entre un conjunto de variables. Lo anterior justifica el hecho de introducir a los estudiantes de grados básicos en un contexto argumentativo que evoque conocimientos sobre fenómenos científicos, pues le permite al docente identificar las relaciones entre las variables. Los discentes a través de este medio pueden evocar sus conocimientos, sea cual fuere su nivel de formación o su dominio del discurso argumentativo.

La intervención educativa, para generar comprensión, parte de la identificación de estos sistemas explicativos con estructuras cognitivas subyacentes, las cuales la ciencia psicológica llama concepciones implícitas (Baldy, 2007). Esto es primordial, pues las concepciones implícitas perduran en el tiempo a pesar de recibir en ocasiones información que las contradice, y la escritura de textos argumentativos sobre fenómenos físicos; en este caso, se ha mostrado fructífera para ponerlas en evidencia.

La escritura de textos sobre fenómenos físicos cotidianos en modalidad colaborativa puede ser una forma propicia para identificar la comprensión que los estudiantes tienen sobre fenómenos físicos al hacerla más evidente para todos los actores del evento 
educativo. Se asume, entonces, que la interacción discursiva que subyace a la escritura de un texto colaborativo puede hacer más explícitas las concepciones implícitas para los mismos estudiantes y generarles, por lo tanto, un desequilibrio, en el sentido piagetiano del término, que genere una transformación de dichas representaciones.

Una de las ventajas del análisis psicolingüístico propuesto es que posibilita tanto estudiar conjuntamente la comprensión de los conocimientos inherentes a la situación expuesta a los estudiantes, como también los recursos lingüísticos que son necesarios para su comunicación. Esta es una de las problemáticas que este artículo intenta enriquecer, pues su finalidad es presentar que la escritura de textos sobre ciencias puede enfocarse desde la comprensión de fenómenos intuitivos que sean interpretables por los niños y las niñas, hacia la implementación de competencias curriculares del área del lenguaje. Así, puede crearse una situación que sea abordable para el niño o la niña desde sus potencialidades y que permita la integración de diversos contenidos disciplinares con un sentido evidente para este. La creación de oportunidades para la implementación, discusión y difusión de contenidos relacionados con ciencia debe ser prioritaria para el correcto desempeño de los niños y las niñas en una sociedad moderna; y la escritura de textos argumentativos puede ser una de estas oportunidades para lograrlo.

La investigación en escritura colaborativa de textos con contenido científico ha indagado la posible interacción entre la demanda cognitiva que genera la apropiación del conocimiento conceptual de las ciencias y la exigencia misma de producir un texto de calidad con todo lo que ello implica. Para Shaw, Van Horne, Zhang y Boughman (2008), la investigación sobre el aprendizaje de la ciencia sugiere que los conceptos erróneos de los estudiantes sobre esta sirven como obstáculos a los estudiantes para alcanzar sus logros. Estos autores, al solicitar la escritura de ensayos sobre genética a estudiantes de noveno a duodécimo grado en Estados Unidos, encontraron que existe una gran dificultad para diferenciar la falta de precisión en la escritura en sí misma de las concepciones erróneas sobre el área de estudio. Aparentemente los discentes no reciben formación sobre lenguaje técnico y parecen aproximarse a la escritura científica de la misma manera en que abordan un ensayo de inglés o de ética. La interacción generada por la escritura colaborativa podría permitirles el enfocarse en el conocimiento necesario para resolver la consigna de la actividad al permitir simultáneamente una redistribución de las acciones y demandas cognitivas que genera la escritura del texto mismo, mejorando así el contenido. 
Este mejoramiento puede encontrase en otro tipo de formatos textuales. Sawmiller (2010) ha propuesto que la escritura colaborativa a través de blogs en el aula de clase puede ayudar a enseñar conceptos científicos y aumentar el aprendizaje mediante el interés personal y el compromiso de un estudiante, fomentando así la autorreflexión y el pensamiento crítico. Chin y Osborne (2010) afirman, por su parte, que, en un ambiente de indagación científica en el aula de clase, el escribir grupalmente preguntas antes de argumentar oralmente modifica sustancialmente la calidad de los argumentos finales. La escritura colaborativa otorga presumiblemente la estructura que ayuda a verbalizar los argumentos.

La idea de base en este artículo entonces es que la interpretación de los fenómenos físicos a través de la escritura de textos científicos puede ser una alternativa que complemente las prácticas educativas que han caracterizado tradicionalmente la educación en ciencias naturales, promoviendo así el razonamiento causal. No se excluyen dichas prácticas, pero, como lo ha puesto de manifiesto Niaz (2010), la reconstrucción histórica de los diferentes temas de la ciencia física muestra que, aunque los experimentos son importantes, la interpretación de los datos lo es aún más. Para este autor, si se quiere que los estudiantes entiendan realmente la práctica científica, es necesario una revisión del plan de estudios de ciencia que les permita tanto analizar cuadros, tablas con resultados numéricos, como también escribir anécdotas de trabajo en laboratorios y escribir, finalmente, informes de investigación. Es allí donde la escritura científica adquiere su importancia al permitirles externalizar su conocimiento.

En este artículo, se ha mostrado que la escritura colaborativa es una forma efectiva de impulsar el pensamiento reflexivo, pues los estudiantes están involucrados en el acto de explicar y defender sus ideas. Ceberio, Almudí y Zubimendi (2014) afirman que el análisis de este tipo de argumentos elaborados puede aportar información importante acerca de la comprensión de los contenidos científicos alcanzada y el razonamiento científico. Por todo ello, y tal y como lo señala Jiménez-Aleixandre (2007), la argumentación debe formar parte de los objetivos de la ciencia constructivista en el aula, en la que los discentes generan conocimiento a través de la reflexión sobre las experiencias del mundo. De igual forma, Özdem, Çavaş, Çavaş, Çakıroğlu y Ertepinar (2010) han remarcado también la importancia de la comunicación y la colaboración dentro de la adquisición de la escritura científica, ya que esta interacción permite la resolución de problemas rutinarios, evocar evidencia que apoye demandas de conocimiento específicas, y genera, a la vez, que los estudiantes 
aprendan a trabajar en equipo buscando formas novedosas de resolver problemas novedosos.

Este mejoramiento ha sido explicado desde diversas aproximaciones teóricas y por diversos procesos, entre ellos el de la motivación. Según Järvelä, Järvenoja y Veermans (2008), las orientaciones motivacionales de los estudiantes entre sí son un factor de mejoramiento en los desempeños grupales. Sus resultados muestran que en una situación de aprendizaje colaborativo, para que sea desafiante socialmente, un miembro del grupo individual debe desempeñar un papel destacado en la activación de regulación de la motivación de todo el grupo. Las tareas de aprendizaje socialmente compartido pueden también estimular nuevas estrategias para la regulación de la motivación.

Se halló en el contexto grupal un mayor número de afirmaciones causales al fenómeno propuesto. Esta aparición de afirmaciones causales se produjo, ya que los estudiantes aportaron sus conocimientos individuales permitiendo que todos los participantes de cada grupo se beneficiaran de la discusión generada. Este mejoramiento es similar a otras investigaciones sobre aprendizaje colaborativo. Noroozi, Biemans, Busstra, Mulder y Chizari (2011) han hallado que los procesos de aprendizaje entre parejas de estudiantes más exitosas y las parejas menos exitosas en términos de construcción de conocimiento difieren significativamente.

El análisis de los argumentos causales compilados en este artículo muestra, por otra parte, que la estructura argumentativa no se complejiza necesariamente por el trabajo colaborativo de escritura. En efecto, el número de afirmaciones causales aumenta porque aumenta el nivel de razonamiento, y nuevas variables explicativas son incorporadas gracias a la interacción entre los participantes cuando se estructura y escribe el texto; sin embargo, la complejidad de los argumentos, que es un indicador de la calidad de los estos, no aumenta.

¿Cómo puede discutirse el problema de la calidad o complejidad de un argumento? La literatura empírica sobre argumentación ha utilizado generalmente el modelo de Toulmin para analizar los datos obtenidos. Para Toulmin (1993), los argumentos tienen una estructura y su validez está ligada a la noción de lógica. El aporte más conocido de este autor al campo de la argumentación ha sido su esquema analítico conocido como "el modelo de Toulmin". Según este, todo argumento formal puede descomponerse en datos que se invocan para apoyar una conclusión, de la cual busca su valor y veracidad; en garantías, las cuales son reglas o principios que validan una inferencia y que permiten el paso de los datos a la 
conclusión y los fundamentos de dichas garantías. El modelo propone, además, un cualificador que hace referencia explícita al grado de fuerza con el cual los datos confirman la conclusión y sus condiciones de refutación. (Gutiérrez y Correa, 2009)

Un indicador de mejoramiento cognitivo desde la teoría de Toulmin y que ha sido explorada con amplitud en diversos estudios (Heng, Surif, y Seng, 2014, 2015; McNeill y Martin, 2011; McNeil y Pimentel, 2010) es la capacidad que tiene una persona para proponer contra-argumentos o refutaciones. En el caso que atañe a las categorías de análisis propuestas en este artículo, los contra-argumentos no se ven modificados por el diseño expuesto en esta investigación. En la amplia serie de trabajos que a la argumentación le ha dedicado Deanna Kuhn (Felton y Kuhn 2001; Kuhn, 1991; Kuhn, lordanou, Pease y Wirkala, 2008; Kuhn y Crowell, 2011; Kuhn, Zillmer, Crowell y Zavala; 2013), se ha concluido que la capacidad de crear argumentos válidos demanda un gran esfuerzo y su dominio se alcanza solo finalizando la adolescencia, y esto debido al tipo de razonamiento epistemológico que la argumentación exige. Por lo tanto, la ausencia de cambios en los textos debido a las condiciones experimentales propuestas demanda a los futuros estudios por realizar sobre la escritura colaborativa, establecer cuál es la intensidad del período de formación en competencias argumentativas y el número de sesiones de escritura colaborativa necesarias para lograr la transformación de los textos de manera argumentativa.

El mejoramiento de la calidad de los textos argumentativos no se alcanza fácilmente, aún en grados superiores. Heng, Surif, y Seng (2014), analizando los textos sobre química escritos por estudiantes de secundaria en Malasia, han propuesto que diferencias significativas pueden hallarse en la producción textual entre el grupo y los individuos, siendo mayor el resultado del grupo. Sin embargo, independientemente de la condición de escritura, el dominio de la argumentación científica para todos los estudiantes no es satisfactorio. Esto es, según los autores, debido en parte a la falta de conocimientos para la correcta justificación de argumentos sobre química. Esto está en sintonía con los resultados aquí presentados. La estructura argumentativa no presenta un mejoramiento más allá de las afirmaciones causales, pues no se ha alcanzado un dominio conceptual y la adquisición de la competencia argumentativa aún está en desarrollo. Con respecto a la relación entre nivel del conocimiento de los estudiantes y su capacidad para argumentar sobre temáticas específicas, Sadler y Donnelly (2006) han afirmado que solamente un alto contenido de conocimiento garantiza un alto nivel de argumentación. 
A pesar de que no se encuentra entonces un mejoramiento sustancial en la argumentación, la necesidad de proveer evidencia que justifique la causalidad propuesta promueve significativamente el análisis de los aspectos del fenómeno discutido. $Y$ esto es un gran aporte del trabajo realizado a través de la escritura de textos científicos, pues les provee experiencias necesarias para poner en prácticas las competencias discursivas ya adquiridas, como también las que están en proceso de desarrollarse. No se propone que los estudiantes de quinto grado (8 a 10 años) carezcan de competencias en la escritura de textos científicos, pero, como se mostró en los resultados, las estructuras macrotextuales son relativamente simples, aunque estables, siendo notable cómo los textos carecen en su mayoría de información que no está circunscrita estrictamente al tópico de discusión. Para autores como Turiman, Omar, Mohd y Kamisah (2012), es necesario incentivar la capacidad de los estudiantes para plasmar en un texto la naturaleza del conocimiento científico, sus teorías y principios. Esto no depende necesariamente de haber alcanzado el dominio de un área disciplinar; los textos recopilados en esta investigación muestran que desde muy pequeños, la maestría de la escritura de textos científicos ha tomado sus primeros cimientos, pues el tópico, las variables y su interacción son consistentes y acordes con los conocimientos formales de la disciplina. Si bien hay que continuar el desarrollo de la capacidad de comunicar ideas de forma escrita, cierto es que se necesitan oportunidades para llevar a feliz término este desarrollo y en este artículo se propone una forma para generarlas.

Esta creación de textos debe guiarse de una consiga sólida que le permita al niño o la niña vincularse rápida y efectivamente con la actividad propuesta. Garcia-Mila, Gilabert, Erduran y Felton (2013) han mostrado que el conocimiento explícito de los objetivos de las tareas que involucran una competencia argumentativa tiene un efecto sobre la calidad de la argumentación de los estudiantes, y ayuda a explicar la forma en que la argumentación mejora los resultados del aprendizaje de las ciencias. Cuando los estudiantes participan en el diálogo argumentativo para llegar a un consenso con un compañero y conocen con claridad qué deben hacer, producen una mayor variedad de estructuras argumentativas, pues existe un problema por resolver conjuntamente y se tiene alguna claridad en torno a si se ha alcanzado o no la meta propuesta. En este sentido, la cercanía con la escritura de textos puede favorecer la comprensión de los objetivos por alcanzar cuando se escribe un texto. 


\section{Conclusiones}

La evidencia de esta investigación da cuenta de diferencias significativas en los textos debido a la interacción de los estudiantes cuando los escriben colaborativamente. Explican sus ideas, discuten argumentativamente y comparten sus concepciones desde diversas perspectivas, reconstruyendo y co-construyendo nuevos conocimientos mientras que solucionan problemas reales. Este estudio revela entonces que la interacción cuando se escribe colaborativamente evoca niveles de razonamiento más complejos en función del número de variables y del tipo de interacción que existe entre ellas. En este estudio la capacidad para reportar secuencialmente en un escrito diversas razones empíricas sobre la aparición de un fenómeno físico parece ser una habilidad presente en el repertorio de los niños y las niñas entre 8 a 10 años de edad. Las estructuras argumentativas no se modifican en todos sus componentes y, por lo tanto, no podría decirse con certitud que la escritura colaborativa de textos mejora sustancialmente la argumentación escrita del estudiantado en el grado analizado en este artículo.

No obstante, el estudio tiene un límite por considerar si se desean generalizar las conclusiones de este estudio. Este es la influencia del Proyecto Educativo Institucional (PEI) en el desarrollo de la escritura y la promoción de la interacción como estrategia pedagógica, lo cual haría disminuir la probabilidad de encontrar resultados similares en otras poblaciones. Se propone un argumento que puede soslayar el posible impacto del PEI en la estrategia colaborativa analizada en el presente artículo: Ciertamente el PEI puede incidir de forma directa la adquisición de la competencia necesaria para escribir textos, pero en esta investigación no se examina normativamente el acto de escritura; en ese sentido, no se discuten niveles de apropiación de la habilidad para escribir, sino las formas en que se comprende el fenómeno físico. Es el razonamiento mismo y no la argumentación causal lo que muestra niveles de complejidad mayor en el contexto colaborativo, siendo esto consistente con los estudios sobre lengua extranjera discutidos ya en los referentes teóricos. De tal forma, se considera que, frente a la escritura colaborativa, este estudio es evidencia de la viabilidad de las propuestas de autores como Dobao (2012), solo que en un área de conocimientos no explorada hasta ahora con plenitud. 


\section{Referencias}

Atance, Cristina, Metcalf, Jennifer, Martin-Ordas, Gema y Walker, Cheryl. (2014). Young children's causal explanations are biased by post-action associative information. Developmental psychology, 50(12), 2675.

Baldy, Elise. (2007). A new educational perspective for teaching gravity. International Journal of Science Education, 29(14), 1767-1788.

Berland, Leema y Lee, Victor. (2012). In pursuit of consensus: Disagreement and legitimization during small-group argumentation. International Journal of Science Education, 34(12), 1857-1882.

Buty, Christian y Plantin, Christian. (2008). Argumenter en classe de sciences. Du débat à l'apprentissage. Paris, France: Institut National de Recherche Pédagogique.

Cavagnetto, Andy, Hand, Brian y Norton-Meier, Lori. (2010). The nature of elementary student science discourse in the context of the science writing heuristic approach. International Journal of Science Education, 32(4), 427-449.

Ceberio, Mikel, Almudí, José Manuel y Zubimendi, José Luis. (2014). Análisis de los argumentos elaborados por estudiantes de cursos introductorios de Física universitaria ante situaciones problemáticas. Enseñanza de las Ciencias, 32, 71-88.

Charaudeau, Patrick. (2009). Identités Sociales et Discursives du Sujet Parlant. Paris: L'Harmattan.

Chin, Christine y Osborne, Jonathan. (2010). Students' questions and discursive interaction: Their impact on argumentation during collaborative group discussions in science. Journal of Research in Science Teaching, 47(7), 883-908.

Colegio Colombiano de Psicólogos, COLPSIC. (2009). Deontología y bioética del ejercicio de la psicología en Colombia. Bogotá, Colombia: Javegraf. Recuperado de http://www.infopsicologica.com/documentos/2009/Deontologia libro.pdf

Coolican, Hugh (2005). Métodos de investigación y estadística en psicología. Bogotá, Colombia: Editorial El Manual Moderno.

Dawson, Vaille y Venville, Grady Jane. (2009). High-school students' informal reasoning and argumentation about biotechnology: An indicator of scientific literacy? International Journal of Science Education, 31(11), 1421-1445.

Dobao, Ana Fernandez. (2012). Collaborative writing tasks in the L2 classroom: Comparing group, pair, and individual work. Journal of Second Language Writing 21, 40-58.

Dobao, Ana Fernández y Blum, Avram. (2013). Collaborative writing in pairs and small groups: Learners' attitudes and perceptions. System, 41(2), 365-378.

Elola, Idoia y Oskoz, Ana. (2010). Collaborative writing: Fostering foreign language and writing conventions development. Language Learning and Technology, 14(3), 51-71. 
Erduran, Sibel, y Jiménez-Aleixandre, María Pilar. (2008). Argumentation in science education. Perspectives from classroom-Based Research. Dordrecht: Springer.

Gadgil, Soniya, Nokes-Malach, Timothy y Chi, Michelene. (2012). Effectiveness of holistic mental model confrontation in driving conceptual change. Learning and Instruction, 22(1), 47-61.

Garcia-Mila, Merce, Gilabert, Sandra, Erduran, Sibel y Felton, Michael. (2013). The effect of argumentative task goal on the quality of argumentative discourse. Science Education, 97(4), 497-523.

Gosser Jr, David, Kampmeier, Jack y Varma-Nelson, Pratibha. (2010). Peer-led team learning: 2008 James Flack Norris award address. Journal of Chemical Education, 87(4), 374-380.

Gutiérrez, Mario Fernando y Correa, Miralba. (2009). Operaciones discursivas y contextos argumentativos: Sobre la comprensión del fenómeno físico de rebotar. Acta Colombiana de Psicología, 12(2), 85-95.

Hardy, Ilonca, Kloetzer, Birgit, Moeller, Kornelia, y Sodian, Beate. (2010). The analysis of classroom discourse: Elementary school science curricula advancing reasoning with evidence. Educational Assessment, 15(3-4), 197-221.

Heng, Lee, Surif, Johari, y Seng, Cher. (2014). Individual versus group argumentation: Student's performance in a Malaysian context. International Education Studies, 7(7), 109-124.

Heng, Lee, Surif, Johari, y Seng, Cher. (2015). Malaysian students' scientific argumentation: do groups perform better than individuals? International Journal of Science Education, 37(3), 505-528.

Järvelä, Sanna, Järvenoja, Hanna y Veermans, Marjaana. (2008). Understanding the dynamics of motivation in socially shared learning. International Journal of Educational Research, 47(2), 122-135.

Jiménez-Aleixandre, Maria Pilar. (2007). Designing Argumentation Learning Environments. En Sibel Erduran y María Pilar Jiménez-Aleixandre (Eds.). Argumentation in science education: perspectives from classroom based research (pp. 91-116). Dordrecht, The Netherlands: Springer.

Kim, Young-Suk. (2015). Language and cognitive predictors of text comprehension: Evidence from multivariate analysis. Child Development, 86(1), 128-144.

Kulatunga, Ushiri, Moog, Richard y Lewis, Jennifer. (2013). Argumentation and participation patterns in general chemistry peer-led sessions. Journal of Research in Science Teaching, 50(10), 1207-1231.

Kuhn, Deanna. (1991). The skills of argument. Cambridge University Press. 
Kuhn, Deanna, lordanou, Kalypso, Pease, Maria y Wirkala, Clarice. (2008). Beyond control of variables: What needs to develop to achieve skilled scientific thinking?. Cognitive Development 23, 435-451.

Kuhn, Deanna y Crowell, Amanda. (2011). Dialogic argumentation as a vehicle for developing young adolescents' thinking. Psychological Science 22(4), 545-552.

Kuhn, Deanna, Zillmer, Nicole, Crowell, Amanda y Zavala, Julia. (2013). Developing norms of argumentation: Metacognitive, epistemological, and social dimensions of developing argumentive competence. Cognition and Instruction, 31(4), 456-496.

Kuhn, Deanna. (2015). Thinking together and alone. Educational Researcher, 44(1), 46-53.

Felton, Mark, y Kuhn, Deanna. (2001). The development of argumentative discourse skill. Discourse Processes, 32(2-3), 135-153.

Leitão, Selma. (2007). Consciência da "estrutura argumentativa" e produção textual. Psicologia: Teoria e Pesquisa, 23(4), 423-432.

Mascalzoni, Elena, Regolin, Lucia, Vallortigara, Giorgio y Simion, Francesca. (2013). The cradle of causal reasoning: newborns' preference for physical causality. Developmental Science, 16(3), 327-335.

Maskiewicz, April Cordero y Lineback, Jennifer Evarts. (2013). Misconceptions are "so yesterday!". CBE-Life Sciences Education, 12(3), 352-356.

McCormack, Teresa, Bramley, Neil, Frosch, Caren, Patrick, Fiona y Lagnado, David. (2016). Children's use of interventions to learn causal structure. Journal of Experimental Child Psychology, 141, 1-22.

McNeill, Katherine y Martin, Dean. (2011). Claims, evidence, and reasoning: Demystifying data during a unit on simple machines. Science and Children, April/May. Recuperado de http://searkscience.pbworks.com/w/file/fetch/70117336/2-Claimsevidence.pdf

McNeill, Katherine y Pimentel, Diane. (2010). Scientific discourse in three urban classrooms: The role of the teacher in engaging high school students in argumentation. Science Education, 94(2), 203-229.

Mercier, Hugo y Sperber, Dan. (2011). Why do humans reason? Arguments for an argumentative theory. Behavioral and Brain Sciences, 34(2), 57-74.

Myhill, Debra, y Jones, Susan. (2015). Conceptualizing metalinguistic understanding in writing/Conceptualización de la competencia metalingüística en la escritura. Cultura y Educación, 27(4), 839-867.

Moeschler, Jacques. (2011). Causal, inferential and temporal connectives: Why parce que is the only causal connective in French. Marqueurs discursifs et subjectivité, 97-114.

Narjaikaew, Pattawan. (2013). Alternative conceptions of primary school teachers of science about force and motion. Procedia - Social and Behavioral Sciences, 88, 250-257. 
Neumann, Heike y McDonough, Kim. (2015). Exploring student interaction during collaborative prewriting discussions and its relationship to L2 writing. Journal of Second Language Writing 27, 84-104.

Niaz, Mansoor. (2010). Science curriculum and teacher education: The role of presuppositions, contradictions, controversies and speculations vs Kuhn's 'normal science'. Teaching and Teacher Education, 26(4), 891-899.

Noroozi, Omid, Biemans, Harm, Busstra, Maria, Mulder, Martin y Chizari, Mohammad. (2011) Differences in learning processes between successful and less successful students in computer-supported collaborative learning in the field of human nutrition and health. Computers in Human Behavior, 27, 309-318

Nussbaum, Michael y Sinatra, Gale. (2003). Argument and conceptual engagement. Contemporary Educational Psychology, 28(3), 384-395.

Özdem, Yasemin, Çavaş, Pinar, Çavaş, Bülent, Çakıroğlu, Jale y Ertepınar, Hamide. (2010). An investigation of elementary students scientific literacy levels. Journal of Baltic Science Education, 9(1), 6-19.

Pereira, Zulay. (2011). Los diseños de método mixto en la investigación en educación: Una experiencia concreta. Revista Electrónica Educare, 15(1), 15-29.

Plantin, Christian. (2014a). Lengua, argumentación y aprendizajes escolares. Tecné, Episteme y Didaxis: Revista de la Facultad de Ciencia y Tecnología, 36, 95-114.

Plantin, Christian. (2014b). Dictionnaire de l'argumentation : Une introduction notionnelle aux études d'argumentation. Lyon, France : ENS Editions.

Pontecorvo, Clotilde. (1993). Forms of discourse and shared thinking. Cognition and Instruction, 11(3 y 4), 189-196.

Sadler, Troy y Donnelly, Lisa. (2006). Socioscientific argumentation: The effects of content knowledge and morality. International Journal of Science Education, 28(12), 1463-1488.

Sawmiller, Alison. (2010). Classroom blogging: What is the role in science learning?. The Clearing House, 83(2), 44-48.

Shaw, Kenna, Van Horne, Katie, Zhang, Hubert y Boughman, Joann. (2008). Essay contest reveals misconceptions of high school students in genetics content. Genetics, 178(3), 1157-1168.

Shehadeh, Ali. (2012). Effects and student perceptions of collaborative writing in L2. Journal of Second Language Writing, 20, 286-305.

Storch, Neomy. (2005). Collaborative writing: Product, process, and students' reflections. Journal of Second Language Writing. 14, 153-173

Storch, Neomy. (2011). Collaborative writing in L2 contexts: Processes, outcomes, and future directions. Annual Review of Applied Linguistics, 31, 275-288. 
Swain, Merrill. (2001). Integrating language and content teaching through collaborative tasks. The Canadian Modern Language Review, 58, 44-63.

Toulmin, Stephen. (1993). Les usages de l'argumentation. Paris: Presses Universitaires de France.

Turiman, Punia, Omar, Jizah, Mohd Daud, Adzliana, y Osman, Kamisah. (2012). Fostering the 21 st century skills through scientific literacy and science process skills. Procedia Social and Behavioral Sciences, 59, 110-116. doi: 10.1016/j.sbspro.2012.09.253

Vygotsky, Liev Semionovich. (1979). El desarrollo de los procesos psicológicos superiores. Buenos Aires: Grijalbo.

Waismeyer, Anna, Meltzoff, Andrew y Gopnik, Alison. (2015). Causal learning from probabilistic events in 24-month-olds: an action measure. Developmental science, 18(1), 175-182.

Walton, Douglas, Reed, Christopher y Macagno, Fabrizio. (2008). Argumentation schemes. Cambridge: Cambridge University Press.

Weisser, Marc. (2004). Compétences argumentatives des enfants d'âge scolaire: Les profils interactionnels au cours préparatoire et au cours moyen. Revue des Sciences de l'Education, 30(2), 435-455.

Wigglesworth, Gillian y Storch, Neomy. (2009). Pairs versus individual writing: effects on fluency, complexity and accuracy. Language Testing, 26, 445-466. 